\title{
Assume-Guarantee Verification for Probabilistic Systems
}

\author{
Marta Kwiatkowska ${ }^{1}$, Gethin Norman ${ }^{2}$, David Parker $^{1}$, and Hongyang $\mathrm{Qu}^{1}$ \\ 1 Oxford University Computing Laboratory, Parks Road, Oxford, OX1 3QD, UK \\ 2 Department of Computing Science, University of Glasgow, Glasgow, G12 8RZ, UK
}

\begin{abstract}
We present a compositional verification technique for systems that exhibit both probabilistic and nondeterministic behaviour. We adopt an assume-guarantee approach to verification, where both the assumptions made about system components and the guarantees that they provide are regular safety properties, represented by finite automata. Unlike previous proposals for assume-guarantee reasoning about probabilistic systems, our approach does not require that components interact in a fully synchronous fashion. In addition, the compositional verification method is efficient and fully automated, based on a reduction to the problem of multi-objective probabilistic model checking. We present asymmetric and circular assume-guarantee rules, and show how they can be adapted to form quantitative queries, yielding lower and upper bounds on the actual probabilities that a property is satisfied. Our techniques have been implemented and applied to several large case studies, including instances where conventional probabilistic verification is infeasible.
\end{abstract}

\section{Introduction}

Many computerised systems exhibit probabilistic behaviour, for example due to the use of randomisation (e.g. in distributed communication or security protocols), or the presence of failures (e.g. in faulty devices or unreliable communication media). The prevalence of such systems in today's society makes techniques for their formal verification a necessity. This requires models and formalisms that incorporate both probability and nondeterminism. Although efficient algorithms for verifying such models are known [318] and mature tool support [117] exists, applying these techniques to large, real-life systems remains challenging, and hence techniques to improve scalability are essential.

In this paper, we focus on compositional verification techniques for probabilistic and nondeterministic systems, in which a system comprising multiple interacting components can be verified by analysing each component in isolation, rather than verifying the much larger model of the whole system. In the case of non-probabilistic models, a successful approach is the use of assume-guarantee reasoning. This is based on checking queries of the form $\langle A\rangle M\langle G\rangle$, with the meaning "whenever component $M$ is part of a system satisfying the assumption $A$, then the system is guaranteed to satisfy property $G$ ". Proof rules can then

J. Esparza and R. Majumdar (Eds.): TACAS 2010, LNCS 6015, pp. 23-37, 2010.
(C) Springer-Verlag Berlin Heidelberg 2010 
be established that show, for example, that if $\langle$ true $\rangle M_{1}\langle A\rangle$ (process $M_{1}$ satisfies assumption $A$ in any environment) and $\langle A\rangle M_{2}\langle G\rangle$ hold, then the combined system $M_{1} \| M_{2}$ satisfies $G$. For probabilistic systems, compositional approaches have also been studied, but a distinct lack of practical progress has been made. In this paper, we address this limitation, presenting the first fully-automated technique for compositional verification of systems exhibiting both probabilistic and nondeterministic behaviour, and illustrating its applicability and efficiency on several large case studies.

We use probabilistic automata 20|21], a well-studied formalism that is naturally suited to modelling multi-component probabilistic systems. Indeed, elegant proof techniques have been developed and used to manually prove correctness of large, complex randomised algorithms [18. Several branching-time preorders (simulation and bisimulation) have been proposed for probabilistic automata and have been shown to be compositional (i.e. preserved under parallel composition) 21, but such branching-time equivalences are often too fine to give significant practical advantages for compositional verification.

A coarser linear-time preorder can be obtained through trace distribution (probability distributions over sequences of observable actions) inclusion 20]; however, it is well known that this relation is not preserved under parallel composition [19. Various attempts have been made to characterise refinement relations that are preserved, e.g. 2015. An alternative direction is to restrict the forms of parallel composition that are allowed. One example is the formalism of switched probabilistic I/O automata [6], which places restrictions on the scheduling between parallel components. Another is [1] which uses a probabilistic extension of Reactive Modules, restricted to synchronous parallel composition. A limitation of all these approaches is that the relations used, such as trace distribution inclusion and weak probabilistic simulation, are not efficiently computable.

We propose an assume-guarantee verification technique for probabilistic automata, that has no restrictions on the parallel composition permitted between components, allowing greater flexibility to model complex systems. To achieve this, we represent both the assumptions made about system components and the guarantees that they provide as safety properties. In the context of probabilistic systems, safety properties capture a wide range of useful properties, e.g. "the maximum probability of an error occurring is at most 0.01 " or "the minimum probability of terminating within $k$ time-units is at least 0.75 ".

We represent safety properties using finite automata and show that verifying assume-guarantee queries reduces to the problem of multi-objective model checking for probabilistic automata [10, which can be implemented efficiently using linear programming. Another key benefit of using finite automata in this way is illustrated by the (non-probabilistic) assume-guarantee verification framework of 16. There, not only is the verification of queries fully automated, but the assumptions themselves (represented as finite automata) are generated automatically using learning techniques. This opens the way for applying learning techniques to compositional verification in the probabilistic case. 
We use our definitions of probabilistic assume guarantee reasoning to formulate and prove several assume-guarantee proof rules, representing commonly occurring patterns of processes. We also discuss how to employ quantitative reasoning, in particular obtaining lower and upper bounds on the actual probability that a system satisfies a safety property. The techniques have been implemented in a prototype tool and applied to several large case studies. We demonstrate significant speed-ups over traditional, non-compositional verification, and successfully verify models that cannot be analysed without compositional techniques.

A full version of this paper, including additional proofs, is available as [12].

Related work. In addition to the compositional techniques for probabilistic systems surveyed above [6115]1819|20|21], we mention several other related pieces of work. In particular, our approach was inspired by the large body of work by Giannakopoulou, Pasareanu et al. (see e.g. [16]) on non-probabilistic assume guarantee techniques. We also build upon ideas put forward in [10], which suggests using multi-objective verification to check probabilistic assumeguarantee queries. Also relevant are: 9], which presents an assume/guarantee framework using probabilistic contracts for non-probabilistic models; 4], which presents a theoretical framework for compositional verification of quantitative (but not probabilistic) properties; and [17, which uses probabilistic automata to model the environment of non-probabilistic components.

\section{Background}

We begin by briefly reviewing probabilistic automata and techniques for their verification. We also introduce safety properties, in the context of probabilistic systems, and discuss multi-objective model checking.

In the following, we use $\operatorname{Dist}(S)$ to denote the set of all discrete probability distributions over a set $S, \eta_{s}$ for the point distribution on $s \in S$, and $\mu_{1} \times \mu_{2} \in$ $\operatorname{Dist}\left(S_{1} \times S_{2}\right)$ for the product distribution of $\mu_{1} \in \operatorname{Dist}\left(S_{1}\right)$ and $\mu_{2} \in \operatorname{Dist}\left(S_{2}\right)$.

\subsection{Probabilistic Automata}

Probabilistic automata 2021] are a modelling formalism for systems that exhibit both probabilistic and nondeterministic behaviour.

Definition 1. A probabilistic automaton $(P A)$ is a tuple $M=\left(S, \bar{s}, \alpha_{M}, \delta_{M}, L\right)$ where $S$ is a set of states, $\bar{s} \in S$ is an initial state, $\alpha_{M}$ is an alphabet, $\delta_{M} \subseteq$ $S \times\left(\alpha_{M} \cup\{\tau\}\right) \times \operatorname{Dist}(S)$ is a probabilistic transition relation and $L: S \rightarrow 2^{A P}$ is a labelling function, assigning atomic propositions from a set AP to each state.

In any state $s$ of a PA $M$, a transition, denoted $s \stackrel{a}{\rightarrow} \mu$, where $a$ is an action label and $\mu$ is a discrete probability distribution over states, is available 1 if $(s, a, \mu) \in \delta_{M}$. In an execution of the model, the choice between the available

${ }^{1}$ Markov decision processes, another commonly used model, are PAs with the restriction that action labels are unique amongst the available transitions for each state. 
transitions in each state is nondeterministic; the choice of successor state is then made randomly according to the distribution $\mu$. A path through $M$ is a (finite or infinite) sequence $s_{0} \stackrel{a_{0}, \mu_{0}}{\longrightarrow} s_{1} \stackrel{a_{1}, \mu_{1}}{\longrightarrow} \cdots$ where $s_{0}=\bar{s}$ and, for each $i \geqslant 0, s_{i} \stackrel{a_{i}}{\longrightarrow} \mu_{i}$ is a transition and $\mu_{i}\left(s_{i+1}\right)>0$. The sequence of actions $a_{0}, a_{1}, \ldots$, after removal of any "internal actions" $\tau$, from a path $\pi$ is called a trace and is denoted $\operatorname{tr}(\pi)$.

To reason about PAs, we use the notion of adversaries (also called schedulers or strategies), which resolve the nondeterministic choices in a model, based on its execution history. Formally an adversary $\sigma$ maps any finite path to a sub-distribution over the available transitions in the last state of the path. Adversaries are defined in terms of sub-distributions because they can opt to (with some probability) take none of the available choices and remain in the current state. For this reason, they are are sometimes called partial adversaries. Occasionally, we will distinguish between these and complete adversaries, in which all the distributions are total.

We denote by $\operatorname{Path}_{M}^{\sigma}$ the set of all paths through $M$ when controlled by adversary $\sigma$, and by $A d v_{M}$ the set of all possible adversaries for $M$. Under an adversary $\sigma$, we define a probability space $\operatorname{Pr}_{M}^{\sigma}$ over the set of paths $\operatorname{Path}_{M}^{\sigma}$, which captures the (purely probabilistic) behaviour of $M$ under $\sigma$.

To reason about probabilistic systems comprising multiple components, we will need the notions of parallel composition and alphabet extension:

Definition 2 (Parallel composition of PAs). If $M_{1}=\left(S_{1}, \bar{s}_{1}, \alpha_{M_{1}}, \delta_{M_{1}}, L_{1}\right)$ and $M_{2}=\left(S_{2}, \bar{s}_{2}, \alpha_{M_{2}}, \delta_{M_{2}}, L_{2}\right)$ are PAs, then their parallel composition, denoted $M_{1} \| M_{2}$, is given by the $P A\left(S_{1} \times S_{2},\left(\bar{s}_{1}, \bar{s}_{2}\right), \alpha_{M_{1}} \cup \alpha_{M_{2}}, \delta_{M_{1} \| M_{2}}, L\right)$ where $\delta_{M_{1} \| M_{2}}$ is defined such that $\left(s_{1}, s_{2}\right) \stackrel{a}{\rightarrow} \mu_{1} \times \mu_{2}$ if and only if one of the following holds:

$-s_{1} \stackrel{a}{\rightarrow} \mu_{1}, s_{2} \stackrel{a}{\rightarrow} \mu_{2}$ and $a \in \alpha_{M_{1}} \cap \alpha_{M_{2}}$

$-s_{1} \stackrel{a}{\rightarrow} \mu_{1}, \mu_{2}=\eta_{s_{2}}$ and $a \in\left(\alpha_{M_{1}} \backslash \alpha_{M_{2}}\right) \cup\{\tau\}$

$-s_{2} \stackrel{a}{\rightarrow} \mu_{2}, \mu_{1}=\eta_{s_{1}}$ and $a \in\left(\alpha_{M_{2}} \backslash \alpha_{M_{1}}\right) \cup\{\tau\}$

and $L\left(s_{1}, s_{2}\right)=L_{1}\left(s_{1}\right) \cup L_{2}\left(s_{2}\right)$.

Definition 3 (Alphabet extension). For any $P A M=\left(S, \bar{s}, \alpha_{M}, \delta_{M}, L\right)$ and set of actions $\Sigma$, we extend the alphabet of $M$ to $\Sigma$, denoted $M[\Sigma]$, as follows: $M[\Sigma]=\left(S, \bar{s}, \alpha_{M} \cup \Sigma, \delta_{M[\Sigma]}, L\right)$ where $\delta_{M[\Sigma]}=\delta_{M} \cup\left\{\left(s, a, \eta_{s}\right) \mid s \in S \wedge a \in \Sigma \backslash \alpha_{M}\right\}$.

We also require the notion of projections. First, for any state $s=\left(s_{1}, s_{2}\right)$ of $M_{1} \| M_{2}$, the projection of $s$ onto $M_{i}$, denoted by $\left.s\right|_{M_{i}}$, is $s_{i}$. We extend this notation to distributions over the state space $S_{1} \times S_{2}$ of $M_{1} \| M_{2}$ in the standard manner. Next, for any path $\pi$ of $M_{1} \| M_{2}$, the projection of $\pi$ onto $M_{i}$, denoted $\pi \uparrow_{M_{i}}$, is the path obtained from $\pi$ by projecting each state of $\pi$ onto $M_{i}$ and removing all the actions not in $\alpha_{M_{i}}$ together with the subsequent states.

Definition 4 (Projections of adversaries). Let $M_{1}$ and $M_{2}$ be PAs and $\sigma$ an adversary of $M_{1} \| M_{2}$. The projection of $\sigma$ onto $M_{i}$, denoted $\left.\sigma\right|_{M_{i}}$, is the adversary on $M_{i}$ where, for any finite path $\pi$ of $M_{i}$ :

$\sigma\left\lceil_{M_{i}}(\pi)(a, \mu)=\sum\left\{\left.\left|\operatorname{Pr}\left(\pi^{\prime}\right) \cdot \sigma\left(\pi^{\prime}\right)\left(a, \mu^{\prime}\right)\right| \pi^{\prime} \in \operatorname{Path}_{M_{1} \| M_{2}}^{\sigma} \wedge \pi^{\prime}\right|_{M_{i}}=\pi \wedge \mu^{\prime} \Upsilon_{M_{i}}=\mu \mid\right\}\right.$. 
Compositional reasoning about PAs, and in particular adversary projections, necessitates the use of partial, rather than complete, adversaries. In particular, even if an adversary $\sigma$ of $M_{1} \| M_{2}$ is complete, the projection $\sigma \uparrow_{M_{i}}$ onto one component may be partial.

\subsection{Model Checking for PAs}

The verification of PAs against properties specified either in temporal logic or as automata has been well studied. In this paper, both the states and transitions of PAs are labelled (with sets of atomic propositions and actions, respectively) and we formulate properties that refer to both types of labels. For the former, we will express properties in linear temporal logic (LTL), and for the latter, we will use safety properties represented by deterministic finite automata.

LTL Verification. For an LTL formula $\psi$, PA $M$ and adversary $\sigma \in A d v_{M}$ :

$$
\operatorname{Pr}_{M}^{\sigma}(\psi) \stackrel{\text { def }}{=} \operatorname{Pr}_{M}^{\sigma}\left\{\pi \in \operatorname{Path}_{M}^{\sigma} \mid \pi \models \psi\right\}
$$

where $\pi \models \psi$ denotes satisfaction according to the standard semantics of LTL. Verifying an LTL specification $\psi$ against $M$ typically involves checking that the probability of satisfying $\psi$ meets a probability bound for all adversaries. This reduces to computing the minimum or maximum probability of satisfying $\psi$ :

$$
\operatorname{Pr}_{M}^{\min }(\psi) \stackrel{\text { def }}{=} \inf _{\sigma \in A d v_{M}} \operatorname{Pr}_{M}^{\sigma}(\psi) \text { and } \operatorname{Pr}_{M}^{\max }(\psi) \stackrel{\text { def }}{=} \sup _{\sigma \in A d v_{M}} \operatorname{Pr}_{M}^{\sigma}(\psi) .
$$

The complexity of this computation is polynomial in the size of $M$ and doubly exponential in the size of $\psi[8$. In practice, the LTL formula $\psi$ is small and, for simple, commonly used cases such as $\diamond a p$ ("eventually ap") or $\square a p$ ("globally $a p ")$, model checking is polynomial [3]. Furthermore, efficient implementations of LTL verification exist in tools such as PRISM [11] and LiQuor [7].

Safety Properties. A regular safety property $A$ represents a set of infinite words, denoted $\mathcal{L}(A)$, that is characterised by a regular language of bad prefixes, finite words of which any extension is not in $\mathcal{L}(A)$. More precisely, we will define a regular safety property $A$ by a (complete) deterministic finite automaton (DFA) $A^{\text {err }}=\left(Q, \bar{q}, \alpha_{A}, \delta_{A}, F\right)$, comprising states $Q$, initial state $\bar{q} \in Q$, alphabet $\alpha_{A}$, transition function $\delta_{A}: Q \times \alpha_{A} \rightarrow Q$ and accepting states $F \subseteq Q$. The DFA $A^{\text {err }}$ defines, in standard fashion, a regular language $\mathcal{L}\left(A^{e r r}\right) \subseteq\left(\alpha_{A}\right)^{*}$. The language $\mathcal{L}(A)$ is then defined as $\mathcal{L}(A)=\left\{w \in\left(\alpha_{A}\right)^{\omega} \mid\right.$ no prefix of $w$ is in $\left.\mathcal{L}\left(A^{\text {err }}\right)\right\}$.

Given a PA $M$, adversary $\sigma \in A d v_{M}$ and regular safety property $A$ with $\alpha_{A} \subseteq \alpha_{M}$, we define the probability of $M$ under $\sigma$ satisfying $A$ as:

$$
\operatorname{Pr}_{M}^{\sigma}(A) \stackrel{\text { def }}{=} \operatorname{Pr}_{M}^{\sigma}\left\{\pi \in \operatorname{Path}_{M}^{\sigma} \mid \operatorname{tr}(\pi)\left\lceil_{\alpha_{A}} \in \mathcal{L}(A)\right\}\right.
$$

where $w \uparrow_{\alpha}$ is the projection of word $w$ onto a subset $\alpha$ of its alphabet. We then define $\operatorname{Pr}_{M}^{\min }(A)$ and $\operatorname{Pr}_{M}^{\max }(A)$ as for LTL above. 

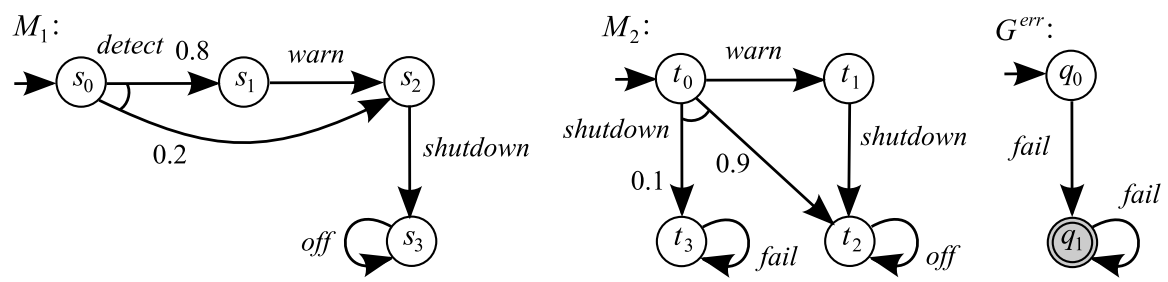

Fig. 1. Two probabilistic automata $M_{1}, M_{2}$ and the DFA for a safety property $G$

Definition 5 (Probabilistic safety properties). A probabilistic safety property $\langle A\rangle_{\geqslant p}$ comprises a regular safety property $A$ and a rational probability bound p. We say that a PA $M$ satisfies the property, denoted $M=\langle A\rangle_{\geqslant p}$, if the probability of satisfying $A$ is at least $p$ for any adversary:

$$
M \models\langle A\rangle_{\geqslant p} \Leftrightarrow \forall \sigma \in A d v_{M} \cdot \operatorname{Pr}_{M}^{\sigma}(A) \geqslant p \quad \Leftrightarrow \quad \operatorname{Pr}_{M}^{\min }(A) \geqslant p .
$$

Safety properties can be used to represent a wide range of useful properties of probabilistic automata. Examples include:

- "the probability of an error occurring is at most 0.01 "

- "event A always occurs before event B with probability at least 0.98 "

- "the probability of terminating within $k$ time-units is at least 0.75 "

The last of these represents a very useful class of properties for timed probabilistic systems, perhaps not typically considered as safety properties. Using the digital clocks approach of 13, verifying real-time probabilistic systems can often be reduced to analysis of a PA with time steps encoded as a special action type. Such requirements are then naturally encoded as safety properties.

Example 1. Figure 1 shows two PAs $M_{1}$ and $M_{2}$. Component $M_{1}$ represents a controller that powers down devices. Upon receipt of the detect signal, it first issues the warn signal followed by shutdown; however, with probability 0.2 it will fail to issue the warn signal. $M_{2}$ represents a device which, given the shutdown signal, powers down correctly if it first receives the warn signal and otherwise will only power down correctly $90 \%$ of the time. We consider a simple safety property $G$ "action fail never occurs", represented by the DFA $G^{\text {err }}$ also shown in Figure 1. Composing the two PAs in parallel and applying model checking, we have that $\operatorname{Pr}_{M_{1} \| M_{2}}^{\min }(G)=0.98$. Thus, $M_{1} \| M_{2} \models\langle G\rangle_{\geqslant 0.98}$.

Safety Verification. Using standard automata-based techniques for model checking PAs [8], verifying correctness of probabilistic safety properties reduces to model checking the product of a PA and a DFA:

Definition 6 (PA-DFA product). The product of a $P A M=\left(S, \bar{s}, \alpha_{M}, \delta_{M}, L\right)$ and $D F A A^{\text {err }}=\left(Q, \bar{q}, \alpha_{A}, \delta_{A}, F\right)$ with $\alpha_{A} \subseteq \alpha_{M}$ is given by the $P A M \otimes A^{\text {err }}=$ $\left(S \times Q,(\bar{s}, \bar{q}), \alpha_{M}, \delta^{\prime}, L^{\prime}\right)$ where:

$-(s, q) \stackrel{a}{\rightarrow} \mu \times \eta_{q^{\prime}}$ if $s \stackrel{a}{\rightarrow} \mu$ and $q^{\prime}=\delta_{A}(q, a)$ if $a \in \alpha_{A}$ and $q^{\prime}=q$ otherwise;

- $L^{\prime}(s, q)=L(s) \cup\left\{\operatorname{err}_{A}\right\}$ if $q \in F$ and $L^{\prime}(s, q)=L(s)$ otherwise. 
Proposition 1. For $P A M$ and regular safety property $A$, we have:

$$
\operatorname{Pr}_{M}^{\min }(A)=1-\operatorname{Pr}_{M \otimes A^{e r r}}^{\max }\left(\operatorname{verr}_{A}\right)
$$

Thus, using [3], satisfaction of the probabilistic safety property $\langle A\rangle_{\geqslant p}$ can be checked in time polynomial in the size of $M \otimes A^{e r r}$. Note that maximum reachability probabilities, and therefore satisfaction of probabilistic safety properties, are independent of whether complete or partial adversaries are considered.

Multi-objective Model Checking. In addition to traditional probabilistic model checking techniques, the approach presented in this paper requires the use of multi-objective model checking [10]. The conventional approach described above allows us to check whether, for all adversaries (or, dually, for at least one adversary), the probability of some property is above (or below) a given bound. Multi-objective queries allow us to check the existence of an adversary satisfying multiple properties of this form. In particular, consider $k$ predicates of the form $\operatorname{Pr}_{M}^{\sigma}\left(\psi_{i}\right) \sim_{i} p_{i}$ where $\psi_{i}$ is an LTL formula, $p_{i} \in[0,1]$ is a rational probability bound and $\sim_{i} \in\{\geqslant,>\}$. Using the techniques in [10], we can verify whether:

$$
\exists \sigma \in A d v_{M} \cdot \wedge_{i=1}^{k}\left(\operatorname{Pr}_{M}^{\sigma}\left(\psi_{i}\right) \sim_{i} p_{i}\right)
$$

by a reduction to a linear programming (LP) problem. Like for (single-objective) LTL verification, this can be done in time polynomial in the size of $M$ (and doubly exponential in the sizes of $\psi_{i}$ ). In fact, [10] also shows that this technique generalises to checking existential or universal queries over a Boolean combination of predicates for which $\sim_{i} \in\{\geqslant,>, \leqslant,<\}$. In all cases, if an adversary which satisfies the predicates exists, then it can also easily be obtained.

Finally, through a trivial extension of this approach (and without increasing the complexity), we can formulate quantitative multi-objective queries. For example, given a conjunction of the above predicates $\Psi=\wedge_{i=1}^{k} \operatorname{Pr}_{M}^{\sigma}\left(\psi_{i}\right) \sim_{i} p_{i}$, and an additional LTL formula $\psi_{0}$, we can compute the maximum probability of $\psi_{0}$ that is achievable whilst also satisfying $\Psi$ :

$$
\left.\operatorname{Pr}_{M}^{\max }\left(\psi_{0} \mid \Psi\right) \stackrel{\text { def }}{=} \sup \left\{\operatorname{Pr}_{M}^{\sigma}\left(\psi_{0}\right) \mid \sigma \in A d v_{M} \wedge \Psi\right)\right\}
$$

\section{Compositional Verification for PAs}

We now describe our approach for compositional verification of probabilistic automata. We first define the basic underlying ideas and then present several different proof rules. For clarity, we present the simplest of these rules in some detail and then discuss some generalisations and extensions.

We extend the notion of assume-guarantee reasoning to PAs using probabilistic assume-guarantee triples of the form $\langle A\rangle_{\geqslant p_{A}} M\langle G\rangle_{\geqslant p_{G}}$, where $\langle A\rangle_{\geqslant} p_{A}$ and $\langle G\rangle_{\geqslant p_{G}}$ are probabilistic safety properties and $M$ is a PA. Informally, the 
meaning of this is "whenever $M$ is part of a system satisfying $A$ with probability at least $p_{A}$, then the system will satisfy $G$ with probability at least $p_{G}$ ". Formally:

Definition 7 (Assume-guarantee semantics). If $\langle A\rangle_{\geqslant p_{A}}$ and $\langle G\rangle_{\geqslant p_{G}}$ are probabilistic safety properties, $M$ is a PA and $\alpha_{G} \subseteq \alpha_{A} \cup \alpha_{M}$, then

$$
\langle A\rangle_{\geqslant p_{A}} M\langle G\rangle_{\geqslant p_{G}} \Leftrightarrow \forall \sigma \in A d v_{M\left[\alpha_{A}\right]} .\left(\operatorname{Pr}_{M\left[\alpha_{A}\right]}^{\sigma}(A) \geqslant p_{A} \rightarrow \operatorname{Pr}_{M\left[\alpha_{A}\right]}^{\sigma}(G) \geqslant p_{G}\right) .
$$

The use of $M\left[\alpha_{A}\right]$, i.e. $M$ extended to the alphabet of $A$, in this definition is required for the case where the property $G$ includes actions that are not in $M$.

We write $\langle$ true $\rangle\langle G\rangle_{\geqslant p_{G}}$ to denote the absence of any assumption, i.e. the query $\langle$ true $\rangle\langle G\rangle_{\geqslant p_{G}}$ is equivalent to $M \models\langle G\rangle_{\geqslant p_{G}}$ which, as described above, is standard model checking [3]. In the general case, we check the satisfaction of a probabilistic assume-guarantee triple using multi-objective PA model checking:

Proposition 2 (Assume-guarantee model checking). Let $M$ be a $P A$, $\langle A\rangle_{\geqslant p_{A}},\langle G\rangle_{\geqslant p_{G}}$ be probabilistic safety properties and $M^{\prime}=M\left[\alpha_{A}\right] \otimes A^{\text {err }} \otimes G^{\text {err }}$. The probabilistic assume-guarantee triple $\langle A\rangle_{\geqslant p_{A}} M\langle G\rangle_{\geqslant p_{G}}$ holds if and only if:

$$
\neg \exists \sigma^{\prime} \in A d v_{M^{\prime}} . \quad\left(\operatorname{Pr}_{M^{\prime}}^{\sigma^{\prime}}\left(\square \neg e r r_{A}\right) \geqslant p_{A} \wedge \operatorname{Pr}_{M^{\prime}}^{\sigma^{\prime}}\left(\nabla e r r_{G}\right)>1-p_{G}\right)
$$

which can be checked in time polynomial in $\left|M^{\prime}\right|$ by solving an LP problem [10]. We now present, using the definitions above, several assume-guarantee proof rules to allow compositional verification.

An asymmetric proof rule. The first rule we consider is asymmetric, in the sense that we require only a single assumption about one component. Experience in the non-probabilistic setting [16] indicates that, despite its simplicity, rules of this form are widely applicable.

Theorem 1. If $M_{1}, M_{2}$ are probabilistic automata and $\langle A\rangle_{\geqslant p_{A}},\langle G\rangle_{\geqslant p_{G}}$ probabilistic safety properties such that $\alpha_{A} \subseteq \alpha_{M_{1}}$ and $\alpha_{G} \subseteq \alpha_{M_{2}} \cup \alpha_{A}$, then the following proof rule holds:

$$
\begin{gathered}
\langle\text { true }\rangle M_{1}\langle A\rangle \geqslant p_{A} \\
\langle A\rangle \geqslant p_{A} M_{2}\langle G\rangle \geqslant p_{G} \\
\hline\langle\text { true }\rangle M_{1} \| M_{2}\langle G\rangle \geqslant p_{G}
\end{gathered}
$$

Theorem 1 means that, given an appropriate assumption $\langle A\rangle_{\geqslant p_{A}}$, we can check the correctness of a probabilistic safety property $\langle G\rangle_{\geqslant p_{G}}$ on $M_{1} \| M_{2}$, without constructing and model checking the full model. Instead, we perform one instance of (standard) model checking on $M_{1}$ (to check the first condition of rule (ASYM)) and one instance of multi-objective model checking on $M_{2}\left[\alpha_{A}\right] \otimes A^{\text {err }}$ (to check the second). If $A^{\text {err }}$ is much smaller than $M_{1}$, we can expect significant gains in terms of the verification performance.

Example 2. We illustrate the rule (ASYM) on the PAs $M_{1}, M_{2}$ and property $\langle G\rangle_{\geqslant 0.98}$ from Example 1. Figure 2 (left) shows a DFA $A^{\text {err }}$ representing the 

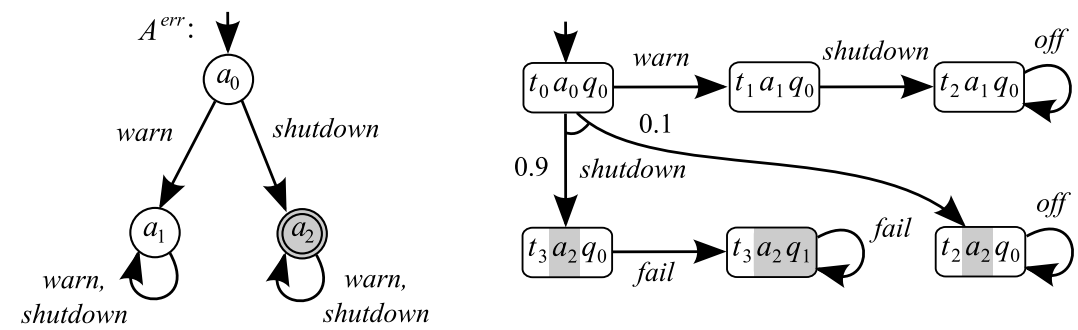

Fig. 2. DFA for safety property $A$ and the product PA $M_{2} \otimes A^{\text {err }} \otimes G^{\text {err }}$ (see Figure 1)

safety property $A$ "warn occurs before shutdown". We will use the probabilistic safety property $\langle A\rangle_{\geqslant 0.8}$ as the assumption about $M_{1}$ in (ASYM).

Checking the first condition of (ASYM) amounts to verifying $M_{1}=\langle A\rangle_{\geqslant 0.8}$, which can be done with standard probabilistic model checking. To complete the verification, we need to check the second condition $\langle A\rangle_{\geqslant 0.8} M_{2}\langle G\rangle_{\geqslant 0.98}$, which, from Proposition 2, is achieved though multi-objective model checking on the product $M_{2} \otimes A^{e r r} \otimes G^{e r r}$. More precisely, we check there is no adversary under which the probability of remaining within states not satisfying $\operatorname{err}_{A}$ is at least 0.8 and the probability of reaching an $\operatorname{err}_{G}$ state is above $1-0.98=0.02$. The product is shown in Figure 2 (right), where we indicate states satisfying err $_{A}$ and $\operatorname{err}_{G}$ by highlighting the accepting states $a_{2}$ and $q_{1}$ of DFAs $A^{\text {err }}$ and $G^{\text {err }}$.

By inspection, we see that no such adversary exists, so we can conclude that $M_{1} \| M_{2} \models\langle G\rangle_{\geqslant 0.98}$. Consider, however, the adversary $\sigma$ which, in the initial state, chooses warn with probability 0.8 and shutdown with probability 0.2 . This satisfies $\square \neg e r r_{A}$ with probability 0.8 and $\nabla e r r_{G}$ with probability 0.02 . Hence, $\langle A\rangle_{\geqslant 0.8} M_{2}\langle G\rangle_{\geqslant p_{G}}$ does not hold for any value of $p_{G}>1-0.02=0.98$.

Proof of Theorem 1. We give below the proof of Theorem 1. This requires the following lemma, which is a simple extension of [20. Lemma 7.2.6, page 141].

Lemma 1. Let $M_{1}, M_{2}$ be PAs, $\sigma \in A d v_{M_{1} \| M_{2}}, \Sigma \subseteq \alpha_{M_{1} \| M_{2}}$ and $i=1,2$. If $A$ and $B$ are regular safety properties such that $\alpha_{A} \subseteq \alpha_{M_{i}}$ and $\alpha_{B} \subseteq \alpha_{M_{i}[\Sigma]}$, then
(a) $\operatorname{Pr}_{M_{1} \| M_{2}}^{\sigma}(A)=\operatorname{Pr}_{M_{i}}^{\sigma \uparrow_{M}}$
and
(b) $\operatorname{Pr}_{M_{1} \| M_{2}}^{\sigma}(B)=\operatorname{Pr}_{M_{i}[\Sigma]}^{\sigma \uparrow_{M_{i}[\Sigma]}}(B)$.

Note that the projections onto $M_{i}[\Sigma]$ in the above are well defined since the condition $\Sigma \subseteq \alpha_{M_{1} \| M_{2}}$ implies that $M_{1}\left\|M_{2}=M_{1}[\Sigma]\right\| M_{2}=M_{1} \| M_{2}[\Sigma]$.

Proof (of Theorem 11). The proof is by contradiction. Assume that there exist PAs $M_{1}$ and $M_{2}$ and probabilistic safety properties $\langle A\rangle_{\geqslant p_{A}}$ and $\langle G\rangle_{\geqslant p_{G}}$ such that $\langle$ true $\rangle M_{1}\langle A\rangle_{\geqslant p_{A}}$ and $\langle A\rangle_{\geqslant p_{A}} M_{2}\langle G\rangle_{\geqslant p_{G}}$ hold, while $\langle$ true $\rangle M_{1} \| M_{2}\langle G\rangle_{\geqslant p_{G}}$ does not. From the latter, it follows that there exists an adversary $\sigma \in A d v_{M_{1} \| M_{2}}$

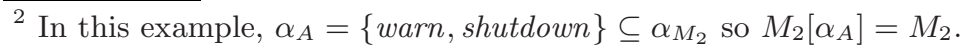


such that $\operatorname{Pr}_{M_{1} \| M_{2}}^{\sigma}(G)<p_{G}$. Now, since $\langle$ true $\rangle M_{1}\langle A\rangle_{\geqslant p_{A}}$ and $\left.\sigma\right|_{M_{1}} \in A d v_{M_{1}}$, it follows that:

$$
\begin{aligned}
& \operatorname{Pr}_{M_{1}}^{\sigma \uparrow_{M_{1}}}(A) \geqslant p_{A} \Rightarrow \operatorname{Pr}_{M_{1} \| M_{2}}^{\sigma}(A) \geqslant p_{A} \quad \text { by Lemma 耳(a) since } \alpha_{A} \subseteq \alpha_{M_{1}} \\
& \Rightarrow \operatorname{Pr}_{M_{2}\left[\alpha_{A}\right]}^{\sigma\left\lceil_{M_{2}\left[\alpha_{A}\right]}\right.}(A) \geqslant p_{A} \text { by Lemma 1, b) since } \alpha_{A} \subseteq \alpha_{M_{2}\left[\alpha_{A}\right]} \\
& \Rightarrow \operatorname{Pr}_{M_{2}\left[\alpha_{A}\right]}^{\sigma \uparrow_{M_{2}\left[\alpha_{A}\right]}}(G) \geqslant p_{G} \quad \text { since }\langle A\rangle_{\geqslant p_{A}} M_{2}\langle G\rangle_{\geqslant p_{G}} \\
& \Rightarrow \operatorname{Pr}_{M_{1} \| M_{2}}^{\sigma}(G) \geqslant p_{G} \quad \text { by Lemma 1(b) since } \alpha_{G} \subseteq \alpha_{M_{2}\left[\alpha_{A}\right]}
\end{aligned}
$$

which contradicts the assumption that $\operatorname{Pr}_{M_{1} \| M_{2}}^{\sigma}(G)<p_{G}$.

Generalising the proof rule. Next, we state two useful generalisations of the above proof rule. First, using $\left\langle A_{1}, \ldots, A_{k}\right\rangle \geqslant p_{1}, \ldots, p_{k}$ to denote the conjunction of probabilistic safety properties $\left\langle A_{i}\right\rangle_{\geqslant p_{i}}$ for $i=1, \ldots, k$, we have:

$$
\begin{gathered}
\langle\text { true }\rangle M_{1}\left\langle A_{1}, \ldots, A_{k}\right\rangle \geqslant p_{1}, \ldots, p_{k} \\
\left\langle A_{1}, \ldots, A_{k}\right\rangle \geqslant p_{1}, \ldots, p_{k} M_{2}\langle G\rangle_{p_{G}}
\end{gathered} \quad \text { (ASYM-Mult) }
$$

Definition 7 extends naturally to $k$ assumptions, replacing $\alpha_{A}$ with $\cup_{i=1}^{k} \alpha_{A_{i}}$ and the single probabilistic safety property on the left-hand side of the implication with the conjunction. In similar fashion, by adapting Proposition 2 , model checking of the query $\left\langle A_{1}, \ldots, A_{k}\right\rangle \geqslant p_{1}, \ldots, p_{k} M\langle G\rangle_{\geqslant p_{G}}$ reduces to multi-objective model checking on the product $M\left[\cup_{i=1}^{k} \alpha_{A_{i}}\right] \otimes A_{1}^{e r r} \otimes \cdots \otimes A_{k}^{e r r} \otimes G^{e r r}$.

Secondly, we observe that, through repeated application of (ASYM), we obtain a rule of the following form for $n$ components:

$$
\begin{gathered}
\langle\text { true }\rangle M_{1}\left\langle A_{1}\right\rangle \geqslant p_{1} \\
\left\langle A_{1}\right\rangle \geqslant p_{1} M_{2}\left\langle A_{2}\right\rangle \geqslant p_{2} \\
\cdots \\
\left\langle A_{n-1}\right\rangle \geqslant p_{n-1} M_{n}\langle G\rangle_{\geqslant p_{G}} \\
\hline\langle\text { true }\rangle M_{1}\|\cdots\| M_{n}\langle G\rangle_{\geqslant p_{G}}
\end{gathered}
$$

(ASYM-N)

A circular proof rule. One potential limitation of the rule (AsYM) is that we may not be able to show that the assumption $A_{1}$ about $M_{1}$ holds without making additional assumptions about $M_{2}$. This can be overcome by using the following circular proof rule:

Theorem 2. If $M_{1}, M_{2}$ are PAs and $\left\langle A_{1}\right\rangle \geqslant p_{1},\left\langle A_{2}\right\rangle \geqslant p_{2}$ and $\langle G\rangle_{\geqslant p_{G}}$ probabilistic safety properties such that $\alpha_{A_{2}} \subseteq \alpha_{M_{2}}, \alpha_{A_{1}} \subseteq \alpha_{M_{1}} \cup \alpha_{A_{2}}$ and $\alpha_{G} \subseteq \alpha_{M_{2}} \cup \alpha_{A_{1}}$, then the following circular assume-guarantee proof rule holds:

$$
\begin{gathered}
\langle\text { true }\rangle M_{2}\left\langle A_{2}\right\rangle \geqslant p_{2} \\
\left\langle A_{2}\right\rangle \geqslant p_{2} M_{1}\left\langle A_{1}\right\rangle \geqslant p_{1} \\
\left\langle A_{1}\right\rangle \geqslant p_{1} M_{2}\langle G\rangle \geqslant p_{G} \\
\hline\langle\text { true }\rangle M_{1} \| M_{2}\langle G\rangle_{\geqslant}
\end{gathered}
$$


An asynchronous proof rule. This rule is motivated by the fact that, often, part of a system comprises several asynchronous components, that is, components with disjoint alphabets. In such cases, it can be difficult to establish useful probability bounds on the combined system if the fact that the components act independently is ignored. For example, consider the case of $n$ independent coin flips; in isolation, we have that the probability of any coin not returning a tail is $1 / 2$. Now, ignoring the independence of the coins, all we can say is that the probability of any of them not returning a tail is at least $1 / 2$. However, using their independence, we have that this probability is at least $1-1 / 2^{n}$.

Theorem 3. For any PAs $M_{1}, M_{2}$ and probabilistic safety properties $\left\langle A_{1}\right\rangle_{\geqslant} p_{A_{2}}$, $\left\langle A_{2}\right\rangle_{\geqslant p_{A_{1}}},\left\langle G_{1}\right\rangle_{\geqslant p_{G_{1}}}$ and $\left\langle G_{2}\right\rangle_{\geqslant p_{G_{2}}}$ such that $\alpha_{M_{1}} \cap \alpha_{M_{2}}=\emptyset, \alpha_{G_{1}} \subseteq \alpha_{M_{1}} \cup \alpha_{A_{1}}$ and $\alpha_{G_{2}} \subseteq \alpha_{M_{2}} \cup \alpha_{A_{2}}$, we have the following asynchronous assume-guarantee proof rule:

$$
\begin{gathered}
\left\langle A_{1}\right\rangle \geqslant p_{A_{1}} M_{1}\left\langle G_{1}\right\rangle \geqslant p_{G_{1}} \\
\left\langle A_{2}\right\rangle \geqslant p_{A_{2}} M_{2}\left\langle G_{2}\right\rangle \geqslant p_{G_{2}} \\
\left\langle A_{1}, A_{2}\right\rangle \geqslant p_{A_{1}}, p_{A_{2}} M_{1} \| M_{2}\left\langle G_{1} \vee G_{2}\right\rangle \geqslant p_{G_{1}}+p_{G_{2}}-p_{G_{1}} \cdot p_{G_{2}}
\end{gathered}
$$

where the disjunction of safety properties $G_{1}$ and $G_{2}$ is obtained by taking the intersection of the DFAs $G_{1}^{\text {err }}$ and $G_{2}^{\text {err }}$.

\section{Quantitative Assume-Guarantee Queries}

Practical experience with probabilistic verification suggests that it is often more useful to adopt a quantitative approach. For example, rather than checking the correctness of a probabilistic safety property $\langle G\rangle_{\geqslant p_{G}}$, it may be preferable to just compute the actual worst-case (minimum) probability $\operatorname{Pr}_{M}^{\min }(G)$ that $G$ is satisfied. In this section we consider how to formulate such quantitative queries in the context of assume-guarantee reasoning. For simplicity, we restrict our attention here to the rule (ASYM) for fixed PAs $M_{1}$ and $M_{2}$, and property $G$. Similar reasoning applies to the other rules presented above.

Maximal lower bounds. Rule (ASYM) allows us to establish lower bounds for the probability $\operatorname{Pr}_{M_{1} \| M_{2}}^{\min }(G)$, i.e. it can be used to prove, for certain values of $p_{G}$, that $\operatorname{Pr}_{M_{1} \| M_{2}}^{\min }(G) \geqslant p_{G}$. We consider now how to obtain the highest such lower bound, say $p_{G}^{\star}$. First, we note that, from Definition 7 , it is clear that the highest value of $p_{G}$ for which $\langle A\rangle_{\geqslant p_{A}} M_{2}\langle G\rangle_{\geqslant p_{G}}$ holds will be obtained by using the maximum possible value of $p_{A}$. For rule (ASym) to be applicable, this is equal to $\operatorname{Pr}_{M_{1}}^{\min }(A)$, since for any higher value of $p_{A}$ the first condition will fail to hold. Now, by Proposition 2 and letting $M^{\prime}=M_{2}\left[\alpha_{A}\right] \otimes A^{e r r} \otimes G^{e r r}$, the value $p_{G}^{\star}$ can be obtained through multi-objective model checking as follows:

$$
p_{G}^{\star}=1-\operatorname{Pr}_{M^{\prime}}^{\max }\left(\diamond e r r_{G} \mid \Psi\right) \text { where } \Psi=\operatorname{Pr}_{M^{\prime}}^{\sigma}\left(\square \neg e r r_{A}\right) \geqslant p_{A} \text {. }
$$


Parameterised queries. Let us assume that component $M_{1}$ is parameterised by a variable $x$ in such a way that varying $x$ changes the probability of $M_{1}$ satisfying the assumption $A$. For example, increasing the value of $x$ might increase the probability $\operatorname{Pr}_{M_{1}}(A)$, but simultaneously worsen some other performance measure or cost associated with $M_{1}$. In this situation, it is desirable to establish a trade-off between the probability of $M_{1} \| M_{2}$ satisfying $G$ and the secondary 'cost' of $M_{1}$. Our use of multi-objective model checking for compositional verification offers two choices here. Firstly, we can pick a suitable threshold for $\operatorname{Pr}_{M_{1} \| M_{2}}(G)$ and then compute the lowest value of $\operatorname{Pr}_{M_{1}}(A)$ which guarantees this, allowing an appropriate value of $x$ to be chosen. Alternatively, we can consider the so-called Pareto curve: the set of achievable combinations of $\operatorname{Pr}_{M_{1} \| M_{2}}(G)$ and $\operatorname{Pr}_{M_{1}}(A)$, which will present a clear view of the trade-off. For the latter, we can use the techniques of [10] for approximate exploration of the Pareto curve.

Upper bounds. Since application of (ASYM) gives lower bounds on $\operatorname{Pr}_{M_{1} \| M_{2}}^{\min }$ $(G)$, it is desirable to also generate upper bounds on this probability. This can be done as follows. When checking condition 2 of (ASYM), using multi-objective model checking, we also obtain an adversary $\sigma \in A d v_{M_{2}\left[\alpha_{A}\right] \otimes A^{\text {err }}}$ that satisfies $\langle A\rangle_{\geqslant p_{A}}$ and gives the minimum (i.e. worst-case) probability of satisfying $G$. This can then be projected onto $M_{2}$, giving an adversary $\sigma_{2}$ which achieves the worstcase behaviour of the single component $M_{2}$ with respect to $G$ satisfying $\langle A\rangle_{\geqslant p_{A}}$. Furthermore, from $\sigma_{2}$, we can easily construct a PA $M_{2}^{\sigma_{2}}$ that represents the behaviour of $M_{2}$ under $\sigma_{2}$.

Finally, we compute the probability of satisfying $G$ on $M_{1} \| M_{2}^{\sigma_{2}}$. Because $M_{2}^{\sigma_{2}}$ is likely to be much smaller than $M_{2}$, there is scope for this to be efficient, even if model checking $M_{1} \| M_{2}$ in full is not feasible. Since $M_{1} \| M_{2}^{\sigma_{2}}$ represents only a subset of the behaviour of $M_{1} \| M_{2}$, the probability computed is guaranteed to give an upper bound on $\operatorname{Pr}_{M_{1} \| M_{2}}^{\min }(G)$. We use $\sigma_{2}$ (which achieves the worst-case behaviour with respect to $G$ ), rather than an arbitrary adversary of $M_{2}$, in order to obtain a tighter upper bound.

\section{$5 \quad$ Implementation and Case Studies}

We have implemented our compositional verification approach in a prototype tool. Recall that, using the rules given in Section 3, verification requires both standard (automata-based) model checking and multi-objective model checking. Our tool is based on the probabilistic model checker PRISM [11, which already supports LTL model checking of probabilistic automata. Model checking of probabilistic safety properties, represented by DFAs, can be achieved with existing versions of PRISM, since DFAs can easily be encoded in PRISM's modelling language. For multi-objective model checking, we have extended PRISM with an implementation of the techniques in 10. This requires the solution of Linear Programming (LP) problems, for which we use the ECLiPSe Constraint Logic Programming system with the COIN-OR CBC solver, implementing a branchand-cut algorithm. All experiments were run on a $2 \mathrm{GHz}$ PC with $2 \mathrm{~GB}$ RAM. Any run exceeding a time-limit of 24 hours was disregarded. 
We demonstrate the application of our tool to two large case studies. The first is the randomised consensus algorithm of Aspnes \& Herlihy [2]. The algorithm allows $N$ processes in a distributed network to reach a consensus and employs, in each round, a shared coin protocol parameterised by $K$. The PA model is based on [14 and consists of an automaton for each process and for the shared coin protocol of each round. We analyse the minimum probability that the processes decide by round $R$. The compositional verification employs $R-2$ uses of the ASYNC rule to return a probabilistic safety property satisfied by the (asynchronous) composition of the shared coin protocols for the first $R-2$ rounds. This is then used as the assumption of an ASYM rule for the subsystem representing the processes.

The second case study is the Zeroconf network configuration protocol [5]. We use the PA model from [13] consisting of two components, one representing a new host joining the network (parameterised by $K$, the number of probes it sends before using an IP address), and the second representing the environment, i.e. the existing network. We consider two properties: the minimum probability that a host employs a fresh IP address and that a host is configured by time $T$. In each case the compositional verification uses one application of the CIRC rule.

Table 1 shows experimental results for these case studies. We present the total time required for both compositional verification, as described in this paper, and

Table 1. Experimental results, comparing with non-compositional verification

\begin{tabular}{|c|c|c|c|c|c|c|c|}
\hline \multirow{2}{*}{\multicolumn{2}{|c|}{$\begin{array}{c}\text { Case study } \\
\text { [parameters] }\end{array}$}} & \multicolumn{3}{|c|}{ Non-compositional } & \multicolumn{3}{|c|}{ Compositional } \\
\hline & & States & Time $(\mathrm{s})$ & Result $^{\dagger}$ & LP size & Time $(\mathrm{s})$ & Result $^{\dagger}$ \\
\hline \multirow{6}{*}{$\begin{array}{c}\text { consensus } \\
\text { (2 processes) } \\
{[R K]}\end{array}$} & \begin{tabular}{|ll}
3 & 2 \\
\end{tabular} & $\overline{5,158}$ & 1.6 & 0.108333 & 1,064 & $\overline{0.9}$ & 0.108333 \\
\hline & 320 & 40,294 & 108.1 & 0.012500 & 1,064 & 7.4 & 0.012500 \\
\hline & 42 & 20,886 & 3.6 & 0.011736 & 2,372 & 1.2 & 0.011736 \\
\hline & 420 & 166,614 & 343.1 & 0.000156 & 2,372 & 7.8 & 0.000156 \\
\hline & $\begin{array}{ll}5 & 2\end{array}$ & 83,798 & 7.7 & 0.001271 & 4,988 & 2.2 & 0.001271 \\
\hline & 520 & 671,894 & 1,347 & 0.000002 & 4,988 & 8.8 & 0.000002 \\
\hline \multirow{6}{*}{$\begin{array}{c}\text { consensus } \\
\text { (3 processes) } \\
{[R K]}\end{array}$} & $\begin{array}{|ll|}3 & 2\end{array}$ & $1,418,545$ & 18,971 & 0.229092 & 40,542 & 29.6 & 0.229092 \\
\hline & 312 & $16,674,145^{*}$ & time-out & & 40,542 & 49.7 & 0.041643 \\
\hline & 320 & $39,827,233^{*}$ & time-out & & 40,542 & 125.3 & 0.024960 \\
\hline & 42 & $150,487,585$ & 78,955 & 0.052483 & 141,168 & 376.1 & 0.052483 \\
\hline & 412 & $1,053,762,385^{*}$ & mem-out & & 141,168 & 396.3 & 0.001734 \\
\hline & 420 & $2,028,200,209^{*}$ & mem-out & - & 141,168 & 471.9 & 0.000623 \\
\hline \multirow{4}{*}{$\begin{array}{c}\text { zeroconf } \\
{[K]}\end{array}$} & 2 & 91,041 & 39.0 & $2.0 \mathrm{e}-5$ & 6,910 & $\overline{9.3}$ & $3.1 \mathrm{e}-4$ \\
\hline & 4 & 313,541 & 103.9 & $7.3 \mathrm{e}-7$ & 20,927 & 21.9 & $3.1 \mathrm{e}-4$ \\
\hline & 6 & 811,290 & 275.2 & $2.6 \mathrm{e}-8$ & 40,258 & 54.8 & $2.5 \mathrm{e}-4$ \\
\hline & 8 & $1,892,952$ & 592.2 & $9.5 \mathrm{e}-10$ & 66,436 & 107.6 & $9.0 \mathrm{e}-6$ \\
\hline zeroconf & 210 & 665,567 & 46.3 & $5.9 \mathrm{e}-5$ & \begin{tabular}{|c|}
62,188 \\
\end{tabular} & 89.0 & $2.1 \mathrm{e}-4$ \\
\hline (time & 214 & 106,177 & 63.1 & $2.0 \mathrm{e}-8$ & $\mid 101,313$ & 170.8 & $8.1 \mathrm{e}-8$ \\
\hline bounded) & $4 \begin{array}{ll}4 & 10\end{array}$ & 976,247 & 88.2 & $3.3 \mathrm{e}+0$ & 74,484 & 170.8 & $3.3 \mathrm{e}+0$ \\
\hline$\left[\begin{array}{ll}K & T\end{array}\right]$ & $4 \quad 14$ & $2,288,771$ & 128.3 & $7.0 \mathrm{e}-5$ & 166,203 & 430.6 & $3.1 \mathrm{e}-4$ \\
\hline
\end{tabular}

* These models can be constructed, but not model checked, in PRISM.

$\dagger$ Results are maximum probabilities of error so actual values are these subtracted from 1. 
non-compositional verification using PRISM (with the fastest available engine). Note that, in each case, we use the quantitative approach described in Section 4 and give actual (bounds on) probabilities computed. To give an indication of the size of the models considered, we give the number of states for the full (noncompositional) models and the number of variables in the LP problems used for multi-objective model checking in the compositional case.

In summary, we see that the compositional approach is faster in the majority of cases. Furthermore, it allows verification of several models for which it is infeasible with conventional techniques. For the cases where compositional verification is slower, this is due to the cost of solving a large LP problem, which is known to be more expensive than the highly optimised techniques used in PRISM. Furthermore, LP solution represents the limiting factor with respect to the scalability of the compositional approach. We expect that improvements to our technique can be made that will reduce LP problem sizes and improve performance. Finally, we note that the numerical values produced using compositional verification are generally good; in fact, for the consensus case study, the bounds obtained are precise.

\section{Conclusions}

We have presented a compositional verification technique, based on assumeguarantee rules, for probabilistic automata. Properties of these models are represented as probabilistic safety properties, and we show how verifying the resulting assume-guarantee queries reduces to the problem of multi-objective model checking. We also show how this can be leveraged to provide a quantitative approach to compositional verification. In contrast to existing work in this area, our techniques can be implemented efficiently and we demonstrate successful results on several large case studies.

There are several interesting directions for future work. In particular, we plan to experiment with the use of learning techniques to automatically produce the assumptions required for compositional reasoning. We also intend to further develop our compositional proof rules and investigate to what extent they are complete. Finally, we plan to expand the range of properties that can be verified, including for example reward-based specifications.

Acknowledgments. The authors are supported in part by EPSRC grants EP/D07956X and EP/D076625 and European Commission FP 7 project CONNECT (IST Project Number 231167). We also gratefully acknowledge several useful discussions with Kousha Etessami.

\section{References}

1. de Alfaro, L., Henzinger, T., Jhala, R.: Compositional methods for probabilistic systems. In: Larsen, K.G., Nielsen, M. (eds.) CONCUR 2001. LNCS, vol. 2154, pp. 351-365. Springer, Heidelberg (2001)

2. Aspnes, J., Herlihy, M.: Fast randomized consensus using shared memory. Journal of Algorithms 15(1) (1990) 
3. Bianco, A., de Alfaro, L.: Model checking of probabilistic and nondeterministic systems. In: Thiagarajan, P.S. (ed.) FSTTCS 1995. LNCS, vol. 1026. Springer, Heidelberg (1995)

4. Chatterjee, K., de Alfaro, L., Faella, M., Henzinger, T., Majumdar, R., Stoelinga, M.: Compositional quantitative reasoning. In: Proc. QEST 2006 (2006)

5. Cheshire, S., Adoba, B., Gutterman, E.: Dynamic configuration of IPv4 link local addresses, http://www.ietf.org/rfc/rfc3927.txt

6. Cheung, L., Lynch, N., Segala, R., Vaandrager, F.: Switched probabilistic I/O automata. In: Liu, Z., Araki, K. (eds.) ICTAC 2004. LNCS, vol. 3407, pp. 494510. Springer, Heidelberg (2005)

7. Ciesinski, F., Baier, C.: Liquor: A tool for qualitative and quantitative linear time analysis of reactive systems. In: Proc. QEST 2006 (2006)

8. Courcoubetis, C., Yannakakis, M.: Markov decision processes and regular events. In: Paterson, M. (ed.) ICALP 1990. LNCS, vol. 443. Springer, Heidelberg (1990)

9. Delahaye, B., Caillaud, B.: A model for probabilistic reasoning on assume/guarantee contracts. Tech. Rep. 6719, INRIA (2008)

10. Etessami, K., Kwiatkowska, M., Vardi, M.Y., Yannakakis, M.: Multi-objective Model Checking of Markov Decision Processes. In: Grumberg, O., Huth, M. (eds.) TACAS 2007. LNCS, vol. 4424, pp. 50-65. Springer, Heidelberg (2007)

11. Hinton, A., Kwiatkowska, M., Norman, G., Parker, D.: PRISM: A tool for automatic verification of probabilistic systems. In: Hermanns, H., Palsberg, J. (eds.) TACAS 2006. LNCS, vol. 3920, pp. 441-444. Springer, Heidelberg (2006)

12. Kwiatkowska, M., Norman, G., Parker, D., Qu, H.: Assume-guarantee verification for probabilistic systems. Tech. Rep. RR-09-17, Oxford University Computing Laboratory (December 2009)

13. Kwiatkowska, M., Norman, G., Parker, D., Sproston, J.: Performance analysis of probabilistic timed automata using digital clocks. Formal Methods in System Design 29 (2006)

14. Kwiatkowska, M., Norman, G., Segala, R.: Automated verification of a randomized distributed consensus protocol using Cadence SMV and PRISM. In: Berry, G., Comon, H., Finkel, A. (eds.) CAV 2001. LNCS, vol. 2102, pp. 194-206. Springer, Heidelberg (2001)

15. Lynch, N., Segala, R., Vaandrager, F.: Observing branching structure through probabilistic contexts. SIAM Journal on Computing 37(4), 977-1013 (2007)

16. Pasareanu, C., Giannakopoulou, D., Bobaru, M., Cobleigh, J., Barringer, H.: Learning to divide and conquer: Applying the L* algorithm to automate assumeguarantee reasoning. Formal Methods in System Design 32(3) (2008)

17. Pavese, E., Braberman, V., Uchitel, S.: Probabilistic environments in the quantitative analysis of (non-probabilistic) behaviour models. In: Proc. ESEC/FSE 2009 (2009)

18. Pogosyants, A., Segala, R., Lynch, N.: Verification of the randomized consensus algorithm of Aspnes and Herlihy: A case study. Dist. Comp. 13(4) (2000)

19. Segala, R.: A compositional trace-based semantics for probabilistic automata. In: Lee, I., Smolka, S.A. (eds.) CONCUR 1995. LNCS, vol. 962, pp. 234-248. Springer, Heidelberg (1995)

20. Segala, R.: Modelling and Verification of Randomized Distributed Real Time Systems. Ph.D. thesis, Massachusetts Institute of Technology (1995)

21. Segala, R., Lynch, N.: Probabilistic simulations for probabilistic processes. Nordic Journal of Computing 2(2) (1995) 